\title{
Predicting Facility-Level Carbapenem-Resistant Enterobacteriaceae (CRE) Incidence Based on Social Network Measures
}

\author{
Michael J. Ray*1, Michael Y. Lin² and William Trick ${ }^{2}$ \\ ${ }^{1}$ Health Policy, Planning, and Statistics, Illinois Department of Public Health, Chicago, IL, USA; ${ }^{2}$ CDC Epicenters Program, Chicago, \\ IL, USA
}

\section{Objective}

To enhance CRE surveillance and communication by incorporating social network measures to quantify patient sharing between facilities.

\section{Introduction}

CRE are multidrug-resistant bacteria associated with up to 50 percent mortality in infected persons (1). CRE are increasingly problematic in Illinois healthcare facilities, especially long-term acute care hospitals (LTACHs); therefore, Illinois implemented the eXtensively Drug-Resistant Organism (XDRO) registry (www.xdro. org). Mathematical models have identified patient sharing between healthcare facilities as a mechanism for regional spread (1), and the importance of each facility within a network can be quantified using social network analysis (2). Degree centrality is a measure representing the number of facilities with which a facility has shared at least one patient, and hence, a measure of "risk" of receiving a CRE colonized patient. Eigenvector centrality is more sophisticated in that it quantifies how well a given node is connected to other "wellconnected" nodes (3). We expect that facilities that have high degree and/or eigenvector centrality - and, thus, higher "risk" of encountering a CRE colonized patient - will have higher incidence of CRE, as will facilities that share patients with LTACHs. Understanding facilitylevel characteristics that predict higher CRE rates will enhance the XDRO registry's usefulness as a surveillance tool.

\section{Methods}

We obtained facility-level CRE cases from the XDRO registry and characteristics from the 2013 Illinois Department of Public Health (IDPH) Annual Hospital Questionnaire. The network analysis was done using IDPH hospital discharge data, and centrality measures were generated using UCINET (Harvard Analytic Technologies 2002). Centrality, number of beds, number of patients shared with LTACHs, and proximity to Chicago were considered as predictors of higher CRE rates. Multivariable negative binomial regression was used to compare incidence rate ratios; we constructed separate models for the state, and stratified by Chicago region.

\section{Results}

Higher centrality and sharing patients with an LTACH was associated with higher CRE rates. These associations hold true for the state and after restricting analysis to the Chicago region (Figure 1). After controlling for number of beds, city proximity, and LTACH sharing, the highest quintile of eigenvector centrality was associated with an over two-fold higher CRE incidence compared to the other quintiles combined (IRR $2.395 \%$ CI: 1.2-4.7); the association remained when analyzing facilities near Chicago (3.0, 1.6-5.4). Degree centrality did not predict a higher CRE rate for the state, but did for the Chicago region (1.5, 1.2-2.1). Sharing patients with LTACHs was a significant predictor in the Chicago eigenvector model $(2.0,1.03-4.0)$ and the statewide degree model $(2.5,1.1-5.8)$.

\section{Conclusions}

There is strong evidence that a facility's centrality and sharing patients with LTACHs predicts higher CRE rates. Combining a surveillance tool such as the XDRO registry with social network measures is a powerful way to understand the geographic spread of antibiotic resistant bacteria. Quantifying patient sharing using the principles of social network analysis allows public health agencies to identify facilities likely to contribute to the spread of antibiotic resistant bacteria, and plan focused interventions.

\section{CRE Rates by Eigenvector Centrality and LTACH Sharing}

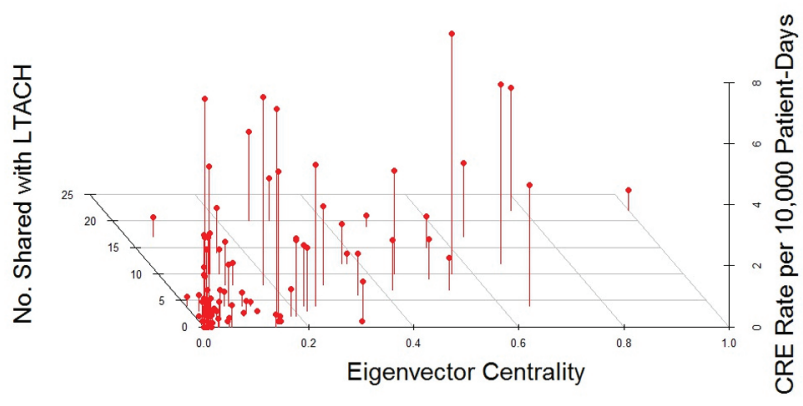

Figure 1: 111 facilties in the Chicago region

\section{Keywords}

Surveillance; Healthcare; Social Network Analysis; HAI

\section{Acknowledgments}

IDPH CRE Taskforce

\section{References}

(1) Centers for Disease Control and Prevention (CDC). (2012) CRE toolkit: guidance for control of carbapenem-resistant Enterobacteriaceae (CRE). Atlanta, Georgia: CDC.

(2) Lee, B., McGlone, S., Song, Y., Avery, T., Eubank, S., Chang, C., Bailey, R., Wagener, D., Burke, D., Platt, R., Huang, S. (2011). Social network analysis of patient sharing among hospitals in Orange County, California. American Journal of Public Health. Vol. 101, No. 4.

(3) Borgatti, S. P., Everett, M. G., \& Johnson, J. C. (2013). Analyzing social networks. SAGE Publications Limited.

\section{*Michael J. Ray}

E-mail: michael.j.ray@illinois.gov 